\title{
COSMIC X-RAY BACKGROUND FROM SELF-ABSORBED LOW LUMINOSITY AGNs
}

\author{
Jonathan E. Grindlay and Michael Luke \\ Harvard/Smithsonian Center for Astrophysics \\ Cambridge, MA U.S.A.
}

\begin{abstract}
A model is proposed for the origin of the diffuse cosmic $x$-ray background whereby it is primarily due to the contribution of low luminosity active galactic nuclei which are increasingly self-absorbed at low luminosities. Strong selfabsorption for low luminosity objects allows the observed background spectrum to be flatter in the $\sim 2-20 \mathrm{keV}$ band than the asymptotic spectrum, assumed to have mean index $\gamma \sim-0.7$ up to a high energy cutoff at $\sim 125 \mathrm{keV}$. The model can account for the spectral shape and intensity of the background spectrum, as well as its possible fluctuations, if the AGN undergo modest density evolution and the bulk of the CXB arises from AGNs at redshifts $z \sim 1-3$. The model can be tested with AXAF observations of low luminosity AGNs at 5-10 keV and sensitive new hard $\mathrm{x}$-ray observations.
\end{abstract}

\section{INTRODUCTION}

The problem of the origin of the diffuse cosmic $x$-ray background (CXB) continues to be one of the major problems of high energy astrophysics. Prompted by the Einstein results for the spectra of AGN and the apparent correlation between $\mathrm{x}$-ray luminosity and low energy absorption (e.g. Lawrence and Elvis 1982), as well as the constraints on the spectrum of the CXB summarized by Boldt (1987), we have developed models for the contribution from low luminosity active galaxies. Although low luminosity AGN have been considered for the CXB (e.g. Giacconi and Zamorani 1987 and references therein), their probable increasingly important self-absorption does not seem to have been considered.

\section{DESCRIPTION OF MODEL}

The basic assumptions of the model are two: that the AGN x-ray spectra are all described by a power law with a luminosity-dependent low energy cutoff and a fixed high energy cutoff, and that the number density of AGN evolve at some rate with cosmic redshift $z$. A third assumption implicit in applying an assumed density evolution is that the form of $x$-ray luminosity function, which increases (in amplitude) with $z$, is just the locally derived value; here we used the parameterization of the luminosity function given by "model a" of Tucker and Schwartz (1986). We 
note that this luminosity function extends with a power law slope of 1.75 down to a low luminosity break (flattening) at $\mathrm{L}_{x} \sim 10^{40.5} \mathrm{erg} / \mathrm{s}$ (cf. also Elvis et al 1984); at luminosities above $10^{43} \mathrm{erg} / \mathrm{s}$, however, it has a steeper slope 3.25 up to an assumed cutoff of $10^{48} \mathrm{erg} / \mathrm{s}$.

The spectral cutoff for low energy absorption (as well as Compton scattering) is assumed to be correlated with x-ray luminosity (scaled to $\sim 10^{43} \mathrm{erg} / \mathrm{sec}$ ) as $\mathrm{E}_{a}$ $=$ const $/ \mathrm{L}_{x}^{\beta}$, where $\mathrm{E}_{a}$ is the cutoff energy related to the absorption and scattering column density by the energy and abundance- dependent relation given in Zombeck (1982), $\mathrm{L}_{x}$ is the luminosity in the Einstein band (0.5-4 keV) and $\beta$ is a parameter of the model. The high energy cutoff is assumed to be fixed at $125 \mathrm{keV}$ (although several trial values in the range $100-300 \mathrm{keV}$ were investigated), as suggested by Schwartz and Tucker (1988) if the cutoff is due to Comptonization of a pair plasma spectrum. The power law index $\gamma$ between the low and high energy cutoffs was taken to be -0.7 in accordance with the mean value found for a broad range of AGN luminosities (e.g. Halpern 1982, Turner and Pounds 1989), although in fact a range of spectral index values $(0.5-0.8)$ were explored in our models. The form of the assumed spectrum and low energy absorption is shown in Figure 1 for the case $\beta$ $=0.5$ and $\gamma=-0.6$ together ( $\mathrm{cf}$. inset) with the data presented by Lawrence and Elvis (1982).

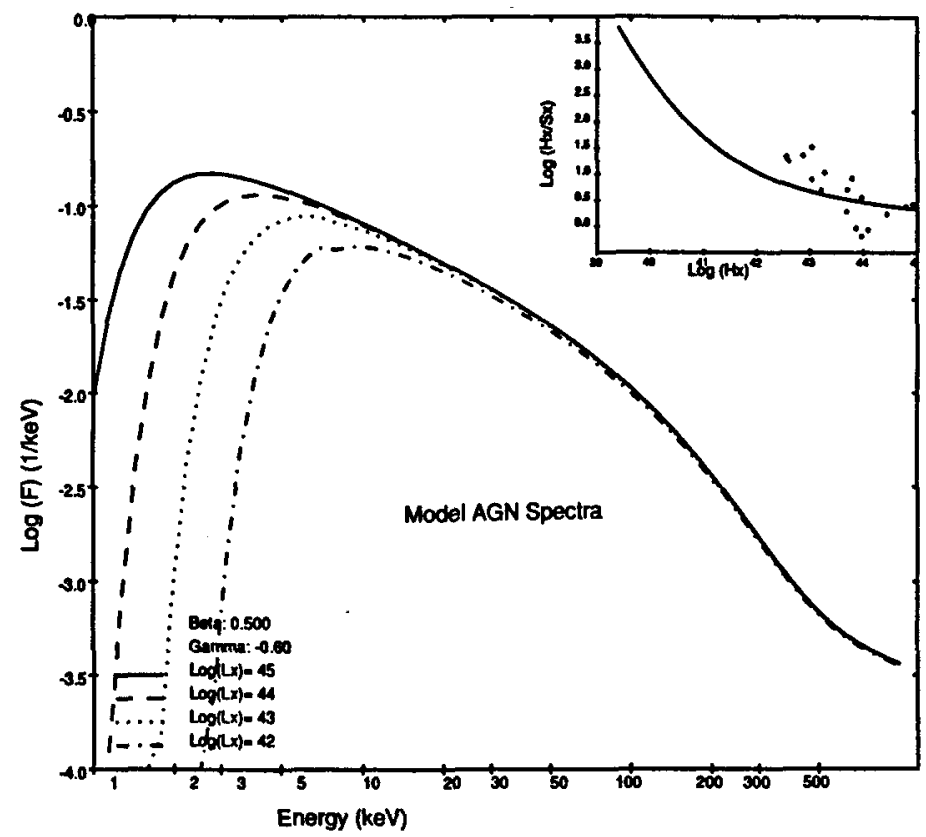

Figure 1: Assumed form of model AGN spectra and cutoffs.

Our key assumption that the low energy cutoff is inversely proportional to the x-ray luminosity is motivated by the results of Lawrence and Elvis (1982) as 
well as the recent body of work on Seyfert IIs (e.g. Antonucci and Miller 1985, Elvis and Lawrence 1988) showing that they contain heavily obscured AGN sources. It is evident from Figure 1 that with an increasingly large contribution from low luminosity AGN, their integrated spectrum will flatten below $\sim 10-20 \mathrm{keV}$.

The assumed density evolution is included in the normalizing constant of the $\mathrm{x}$-ray luminosity function $\mathrm{N}_{o, z}=\mathrm{N}_{o} \cdot e^{(\alpha \cdot \tau)}$, where $\alpha$ is the second parameter of the model and $\tau$ is the 'lookback' time and is $\tau=0.67\left(1-[1+z]^{-3 / 2}\right)$.

\section{RESULTS}

Given these assumptions, we fit the residual CXB spectrum of Boldt (1987), which is the spectrum after the contributions from galaxy clusters and bright QSOs are subtracted (which together account for less than $30 \%$ of the flux), over the range 3-60 keV. The results are shown in Figure 2. The inset in the Figure shows the percentage contribution (out to an assumed maximum redshift $z=3.5$ ) to the background at two observed energies; most of the background comes from low luminosity AGN beyond $\mathrm{z}=1$.

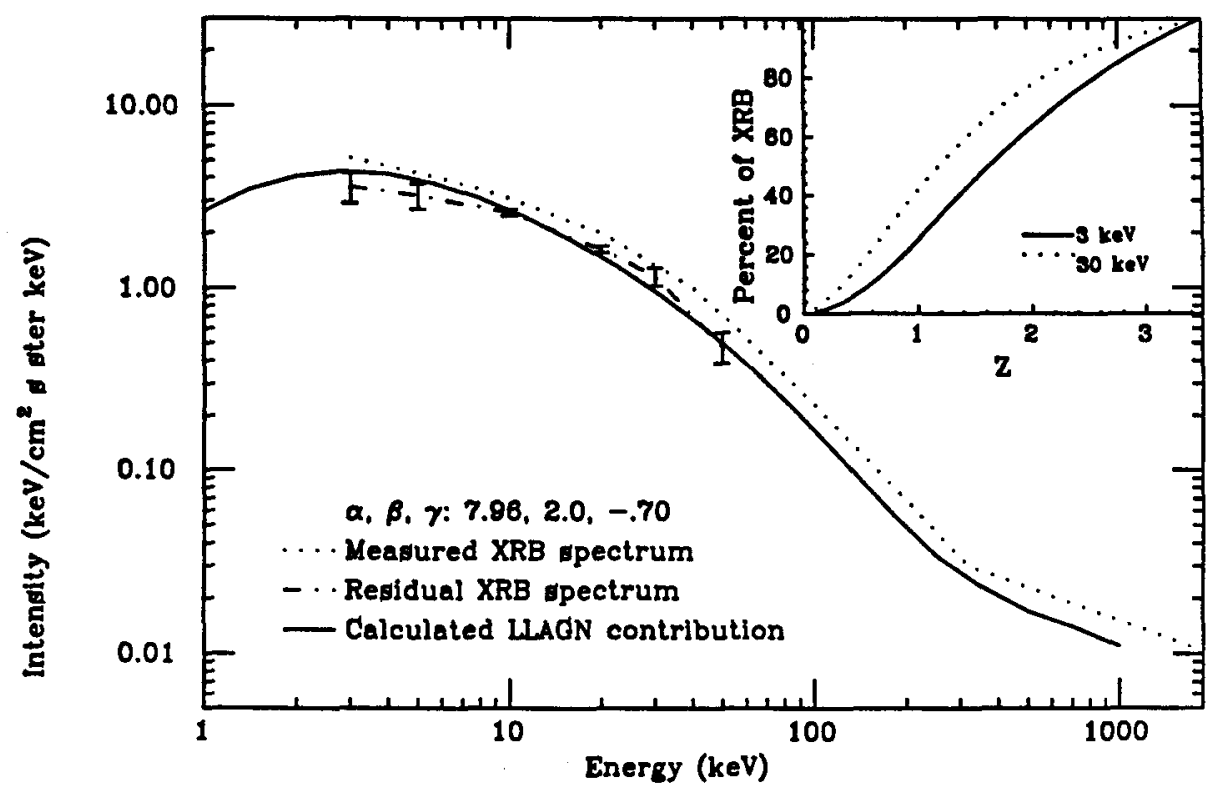

Figure 2: Fit of model to the CXB spectra.

The overall spectral shape and normalization of the CXB spectrum can be fit rather well in the $\mathrm{x}$-ray band (2-100 $\mathrm{keV})$. This model for the CXB can connect to the high energy background spectrum ( $\geq 100 \mathrm{keV}$ ) if we also assume (as in Figure 1) 
that the intrinsic AGN spectra contain a non-thermal power law component with the same spectral slope (e.g. - 0.7 ) but with only 0.1 the flux and a gamma ray cutoff at $\sim 1 \mathrm{MeV}$. This component could naturally arise from the inverse Compton or SSC models of Band and Grindlay (1986).

The "best-fit" values of the parameters $\alpha \simeq 7 \pm 1$ and $\beta \simeq 2 \pm 2$ are such that the required evolution $(\alpha)$ is modest and the required low energy absorption $(\beta)$ is consistent with individual spectra. In Figure 3 we show the contours derived from $\chi^{2}$ fits between the models and the CXB spectrum (Boldt 1987) for the allowed ranges of the parameters $\alpha$ and $\beta$ for three different choices of the spectral index $\gamma$.

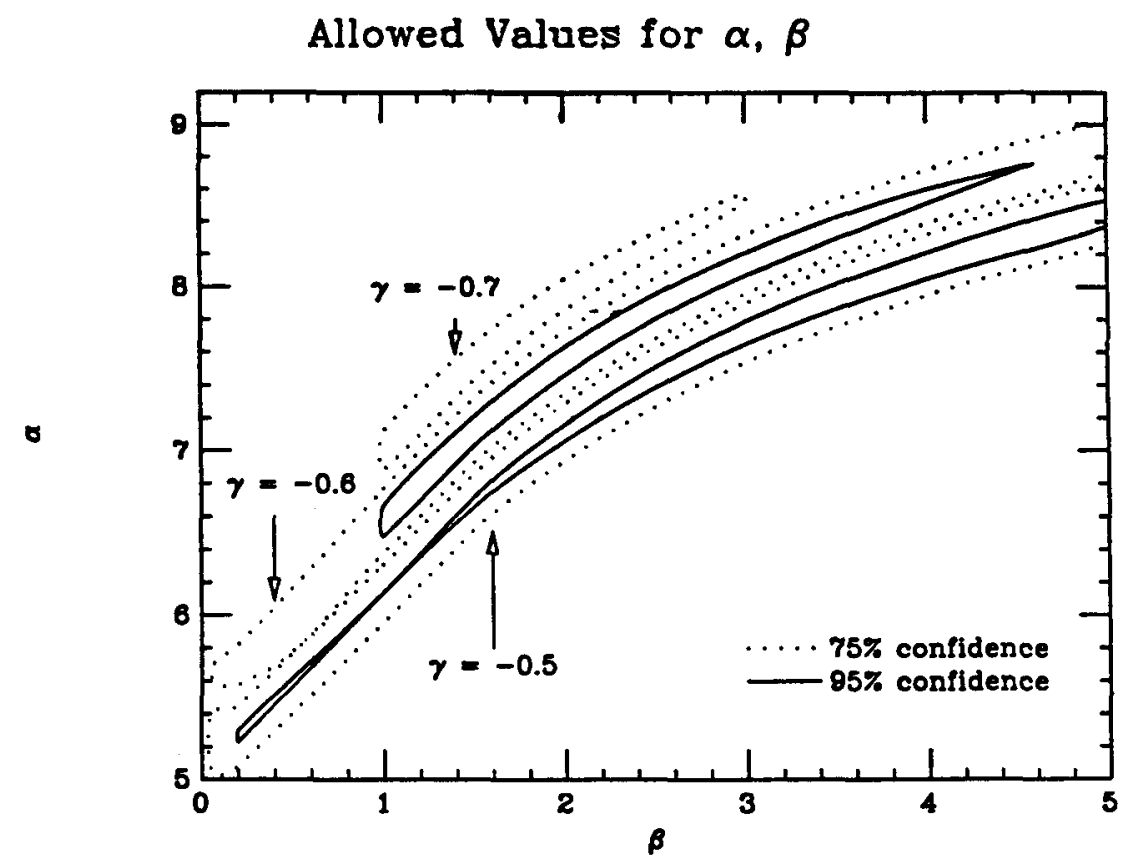

Figure 3: Allowed values for model parameters $\alpha$ and $\beta$.

We note that the range of $\beta$ values indicated would differ for a different choice of scaling (other than the assumed $10^{43} \mathrm{erg} / \mathrm{s}$ ) in our assumed $\mathrm{E}_{a}$ vs. $\mathrm{L}_{x}$ relation. This could be determined from observations but will require complete samples with good $\mathrm{x}$-ray spectra.

The fact that the CXB is primarily due to low luminosity AGN $\left(<10^{43}\right.$ $\mathrm{erg} / \mathrm{sec}$ ) in this model also means that the fluctuation limits (Hamilton and Helfand 1987, Barcons and Fabian 1988) of $\sim 3000$ sources degree ${ }^{-2}$ are easily satisfied.

\section{CONCLUSIONS}

The CXB and possibly also diffuse $\gamma$-ray background spectrum can be fit 
satisfactorily by the integrated effects of self-absorbed, low luminosity AGN. A clear cut test of this model can be made by measuring the spectra of low luminosity AGNs with AXAF. The greatly increased sensitivity and spectral coverage up to nearly 10 $\mathrm{keV}$ will allow a search for the expected low energy absorption $(\leq 10 \mathrm{keV})$ and power law spectra for a large sample of the lowest luminosity $\left(\sim 10^{41} \mathrm{erg} / \mathrm{sec}\right)$ AGN. The spectral resolution with AXAF will also allow the expected low energy absorption vs. scattering effects to be measured. Another test would be observations of many AGNs out to $\geq 100 \mathrm{keV}$ to search for the expected high energy break (which is redshifted down to the observed $\sim 25 \mathrm{keV}$ break). This is a principal objective of coded aperture imaging hard x-ray telescopes, such as EXITE (Grindlay et al 1986), currently under development.

\section{ACKNOWLEDGEMENTS}

We thank M. Elvis and D. Schwartz for comments. This work was supported in part by grants NAGW-624 and NAS 8-30751.

\section{REFERENCES}

Antonucci, R. and Miller J. 1985, Ap. J., 297, 621.

Barcons, X. and Fabian, A. 1988, MNRAS, 290, 189.

Band, D. and Grindlay, J. 1986, Ap. J., 308, 576.

Boldt, E. 1987, Physics Reports, 146, No. 4.

Elvis, M., Soltan, A. and Keel, W. 1984, Ap.J., 283,479.

Elvis, M. and Lawrence, A. 1988, Ap. J., 331, 161.

Giacconi, R. and Zamorani, G. 1987, Ap.J., 312, 503.

Grindlay, J., Garcia, M., Burg, R. and Murray, S. 1986, Trans. Nucl. Sci., 33, No. 1,750 .

Halpern, J. 1982,Ph. D. Thesis, Harvard University.

Hamilton, T. and Helfand, D. 1987, Ap. J., 313, 20.

Lawrence, A. and Elvis, M. 1982, Ap. J., 256, 410.

Schwartz, D. and W. Tucker, W. 1988, Ap. J., 332, 157.

Turner, M. and Pounds, K., 1989, MNRAS, in press.

Tucker, W. and Schwartz, D. 1986, Ap. J., 308, 53.

Zombeck, M. 1982, Handbook of High Energy Astrophysics, Cambridge University Press, Cambridge. 